\title{
Die Verantwortung der
}

\section{Krankenkassen für Sicherstellung}

und Organisation der

gesundheitlichen Versorgung

CHRISTOPHER HERMANN, JÜRGEN GRAF

Dr. Christopher Hermann ist Vorstandsvorsitzender der AOK-Baden-Württemberg

Jürgen Graf ist Fachbereichsleiter „Integriertes Leistungsmanagement" bei der AOK-

Baden-Württemberg

Seit Jahren wird von unterschiedlichster Seite die Segmentierung der gesundheitlichen Versorgung in mehr oder weniger abgeschotteten Sektoren mit jeweils eigener Steuerungs- und Handlungslogik beklagt und auf die negativen ökonomischen und medizinischen Auswirkungen verwiesen. Im Gegenzug wird eine stärkere Integration der Leistungserbringung gefordert. Der vorliegende Beitrag zeigt Lösungsoptionen auf, die auf einer erweiterten Kompetenz- und Verantwortungszuschreibung an Krankenkassen innerhalb eines sozialstaatlich ausgerichteten wettbewerblich geprägten Ordnungsrahmens beruhen. Anhand der Erfahrungen aus den Selektivverträgen der AOK Baden-Württemberg werden Entwicklungsperspektiven aufgezeigt und die entsprechenden politisch gesetzgeberischen Notwendigkeiten benannt.

\section{Status quo: Organisierte Unverantwortlichkeit}

Wie schnell ist nichts passiert? Nicht erst seit dem wegweisenden Gutachten 2000/2001 des Sachverständigenrats zur Begutachtung der Entwicklung im Gesundheitswesen (SVR) unter dem Motto „Bedarfsgerechtigkeit und Wirtschaftlichkeit" (Über-Unter-Fehlversorgung) sowie der nachfolgenden Gutachten mit den Schwerpunkten „Koordination und Qualität“, „Kooperation und Verantwortung“, „Koordination und Integration“ bis zum jüngsten Gutachten „Wettbewerb an der Schnittstelle zwischen ambulanter und stationärer Gesundheitsversorgung“ ist klar, dass
Organisation und Strukturen des deutschen Gesundheitswesens sich nicht ausreichend an den gewandelten Erfordernissen einer Bevölkerung mit steigenden Prävalenzen chronischer Krankheiten in einer Gesellschaft des längeren Lebens ausgerichtet haben.

Die Organisation des Gesundheitswesens ist geprägt von heterogenen Verantwortlichkeiten und Organisationsstrukturen, die letztlich einer notwendigen an den Patienten ausgerichteten effektiven und effizienten Versorgungsstrukturierung entgegen stehen. Im Ergebnis kann das heutige Organisationspuzzle demzufolge mit hoher Berechtigung als „organisierte Verantwortungslosigkeit" bezeichnet werden (vgl. etwa Cassel 2008). 
In der Praxis führt die gegenwärtige Organisation des deutschen Gesundheitswesens aus Patientensicht in eine Situation, dass der medizinische Einsatz häufig nicht von einem bestimmten Behandlungsziel her konkret definiert wird, sondern vielmehr aus dem Einsatz der gerade vorhandenen Ressourcen resultiert („Wer als Werkzeug nur einen Hammer hat, sieht in jedem Problem einen Nagel“).

Diese Grunderkenntnis kontrastiert mit zwei nachhaltigen Entwicklungen. Zum einen der zunehmenden Patientensouveränität mittels einfach zugänglicher Informationen über das Internet sowohl hinsichtlich der Behandlungsoptionen als auch über Bewertungen von Leistungsanbietern. Zum anderen der Notwendigkeit der Versorgungsstrukturierung und der technisch-medizinischen Möglichkeiten für Wissensmanagement und vernetzter Behandlung.

Um diese aus individueller Patientensicht nutzenstiftend anzuwenden, bedarf es gleichzeitig einer hohen Wertigkeit für Beratung und Koordination auf der Grundlage verlässlicher bzw. auf Evidenz basierter Informationen. Um entsprechende strukturierte Behandlungsabläufe über die arbeitsteiligen Sektoren hinweg verlässlich $\mathrm{zu}$ organisieren, müssen diese jedoch aufeinander abgestimmt, um effektive Koordinierungsfunktionen ergänzt und im Zusammenspiel mit nicht ärztlichen Gesundheitsberufen flexibel austariert werden. Auf der „Mikroebene“ wird der einzuschlagende Versorgungspfad im Ergebnis häufig in Abhängigkeit von individuellen Patientenpräferenzen zu definieren sein - wohlgemerkt auf der Grundlage verlässlicher Information und umfassender gemeinsamer Beratung mit dem (Haus-)Arzt.

\section{Effizienz und Steuerungsfähigkeiten}

Nachfolgend sollen zunächst die verschiedenen Effizienzebenen in Erinnerung gebracht werden, da daran auch die unterschiedlichen Handlungsebenen und Steuerungsimplikationen ablesbar sind. Der SVR (2012, S. 37 ff.) unterscheidet im Wesentlichen drei Effizienzebenen. Zunächst - klassisch gesundheitsökonomisch - die allokative Effizienz, die das Verhältnis von gesundheitlichen Outcomes und volks- wirtschaftlichen Kosten betrachtet.

Auf dieser Ebene finden zumeist Gesundheitssystemvergleiche statt (etwa OECD). Hierbei belegt das deutsche Gesundheitswesen zumeist nur mittlere Plätze.

Die nächste Ebene bildet die sog. Produktionseffizienz ab, die sich aus dem Verhältnis des Behandlungsangebots und den volkswirtschaftlichen Kosten

\section{Das deutsche Gesundheits- wesen hat eine hohe Produktionseffizienz: Für viel Geld gibt es sehr viele Leistungen.}

ergibt. Ableiten lässt sich hieraus wohl, dass das deutsche Gesundheitssystem mit umfassendem Leistungskatalog, grundsätzlich freiem Zugang aller Bürgerinnen und Bürger sowie sehr hohen Leistungsraten (z.B. gemessen an Prozeduren je 1.000 Einwohner) eine sehr hohe Produktionseffizienz besitzt oder schlicht: Für viel Geld gibt es auch sehr viele Leistungen.

In einer dritten Ebene wird die Produktionseffizienz in eine finanzielle und eine physische differenziert. Die finanzielle Produktionseffizienz bewertet die unmittelbare Wirtschaftlichkeit (Preisebene), die physische Produktionseffizienz das Behandlungsangebot im Sinne der Frage nach dem optimalen Einsatzverhältnis der verschiedenen Produktionsverfahren (z.B. Prävention, Beratung, Operationen, Rehabilitation, Hygiene, Pflege usw.). Dies impliziert auch Fragen etwa danach, ob die richtigen Leistungen bzw. Verfahren an der richtigen Stelle in der notwendigen Qualität und mit ausreichender Koordinierung eingesetzt werden. Man kann wohl davon ausgehen, dass die so definierte physische Produktionseffizienz bzw. genau dieses Einsatzverhältnis in hohem Maße die letztlich für ein Gesundheitswesen maßgebliche Größe der gesundheitlichen Outcomes beeinflusst.

\section{Ordnungsrahmen}

Hierbei schließt sich der Kreis zur Notwendigkeit, eine übergreifende Verantwortlichkeit und Steuerungsfähigkeit zu etablieren. Wer könnte diese Funktion wahrnehmen? Unbestritten kommt den
Bundesländern eine verfassungsgerichtlich untermauerte politische Verantwortung für die Sicherstellung einer bedarfsgerechten gesundheitlichen Versorgung der Bevölkerung zu, die anhand von Gesundheitszielen bzw. Indikatoren auf der Makroebene stets zu überwachen ist. Diese übergreifende Sicherstellungsverantwortung sollte sich allerdings im Wesentlichen auf eine Aufsichtspflicht über die unmittelbaren Akteure sowie reine Rahmenplanungsvorgaben beschränken. Eine derart vordergründige „Selbstbeschränkung des Staates" auf die konsequente Etablierung und Überwachung eines geeigneten Ordnungsrahmens kann als Konzept zur Stärkung des Staates in einer polyzentrischen $\mathrm{Ge}$ sellschaft verstanden werden, in der er andernfalls Gefahr läuft, sich in mikroökonomischer Beteiligung am pluralistischen Interessenkampf aufzureiben und damit letztlich in ein Legitimitätsdefizit zu geraten. Die konkrete Ausgestaltung der Versorgung sollte vielmehr den Akteuren der Krankenkassen einerseits und der Leistungsanbieter anderseits in einem sozialstaatlich ausgerichteten grundsätzlich wettbewerblich geprägten Ordnungsrahmen überlassen werden.

\section{Krankenkassen und Versorgungssteuerung}

Für die dargelegte notwendige Steuerungsfunktion im Sinne einer effizienten Versorgungsstrukturierung insgesamt scheinen aufgrund ihrer inneren Organisationsstruktur einzig Krankenkassen geeignet, diese übergreifende Verantwortung wahrzunehmen. Während etwa Krankenhausgesellschaften, Kassenärztliche Vereinigungen (KVen) oder Apothekerverbände natürlicherweise insbesondere individuelle Interessen verfolgen und damit nicht zwingend auf eine Gemeinwohlorientierung programmiert sind, stellt sich dies für Krankenkassen anders dar: Der sozialpartnerschaftliche Selbstverwaltungscharakter der Krankenkassen spiegelt im Ergebnis sowohl die Zahler- als auch die Patientenperspektive wider und leistet bereits einen internen Interessenausgleich zwischen möglichst effizientem Mitteleinsatz und möglichst effektiver Versorgung. Unter den Bedingungen des freien Versichertenwettbewerbs i. V. m. dem morbiditätsorientierten Ri- 
sikostrukturausgleich (mRSA) steht das Handeln von Krankenkassen im Sinne dieses Interessenausgleichs unter unmittelbarer Sanktionsdrohung durch Kündigung und letztlich Insolvenz, ohne dass das solidarische System der GKV dadurch insgesamt gefährdet würde. Es sei ohne weitere inhaltliche Ausführungen hier nur angemerkt, dass für eine optimale Funktionsfähigkeit des Wettbewerbs im Sinne von vor allem guter Versorgungslösungen der mRSA bzw. Mechanismen seiner Ausgestaltung weiter anzupassen sind (vgl. Drösler 2011).

Krankenkassen sind die einzigen Akteure im Gesundheitswesen, die grundsätzlich zu allen Segmenten von Leistungsanbietern direkte Vertragsbeziehungen aufweisen, freilich mit sehr unterschiedlichen Regelungsintensitäten und Steuerungsmöglichkeiten. Damit sind Krankenkassen nicht nur hinsichtlich der Legitimität, sondern auch ganz praktisch die einzigen Stakeholder, die prinzipiell die Voraussetzungen erfüllen (können), die verschiedenen Leistungssegmente im Sinne eines gestuften und rationalen Versorgungsprozesses aufeinander abzustimmen.

\section{Politische \\ Handlungsanforderungen}

Verfolgt man dieses Grundkonzept im Weiteren, ergeben sich daraus zunächst insbesondere nachfolgende Anforderungen an Politik und Gesetzgeber:

1) Die solidarisch aufgebrachten Finanzmittel (mRSA) können nur in einem solidarisch einheitlichen Leistungskatalog verwendet werden. Darüber hinausgehende Leistungen sind ggf. konsequent über individuell finanzierte

\section{Krankenkassen sind die einzigen Akteure im Gesundheitswesen, die zu allen Segmenten von Leistungsanbietern direkte Vertragsbeziehungen aufweisen.}

2) Die gesetzlichen Vorgaben $\mathrm{zu}$ einheitlich und gemeinsamen Vertragsabschlüssen von Krankenkassen mit Leistungsanbietern werden konsequent zurückgeführt, insbesondere im Bereich der vertragsärztlichen Versorgung. Die ambulant ärztlichen Teilleistungsbereiche Hausärztliche Versorgung, Fachärztliche Versorgung, Spezialärztliche Versorgung einschließlich des ambulanten Operierens, Psychotherapeutische Versorgung sowie Notfallversorgung (Notdienst) werden in eigenständige Verhandlungs- und Vergütungssäulen gefasst. Allein die Notfallversorgung ist krankenkassenübergreifend einheitlich zu verhandeln und $\mathrm{zu}$ gestalten.

3) Kontrahierungszwänge der Krankenkassen mit Leistungsanbietern werden neu geordnet, insbesondere Aufhebung für fachärztliche und spezialfachärztliche Leistungen sowie für bestimmte v.a. elektive stationäre Leistungen (vgl. etwa Schönbach 2011, Leber 2007).

4) Weiterentwicklung des Zulassungsrechts, etwa durch Ermöglichung fachgebietsgleicher medizinischer Versorgungszentren (MVZ) - z.B. rein hausärztlich - und Vergabe eines neuen Zulassungsstatus', der unter rein qualitativen Prüfkriterien auch eine ausschließliche Teilnahme an Selektivverträgen ermöglicht.

5) Weiterentwicklung und Vereinfachung der Bereinigungsregelungen im Bereich der vertragsärztlichen Versorgung (vgl. etwa Graf 2010, SVR 2012 S.320 ff.) sowie analoge Regelungen für den stationären Bereich.

6) Die Aufgaben der Selbstverwaltungspartner auf Bundesebene, insbesondere DKG, KBV und GKV-SV werden konsequent auf eine Rahmengestaltung zurückgeführt, die insbesondere keine direkten Vergütungsvorgaben enthält und strikt wettbewerbsneutral auf Ebene der Krankenkassen ausgerichtet ist. Die „Ideologie“ gleicher Preise für gleiche Leistungen in ganz Deutschland wird auf-

Wahl- bzw. Zusatztarife anzubieten. Hieraus ergeben sich auch Perspektiven einer Neuordnung bzw. Integration von PKV und GKV (vgl. etwa Greß 2009). Der solidarisch einheitliche Leistungskatalog wird vom Gemeinsamen Bundesausschuss (GBA) definiert. gegeben, da sie insbesondere nicht kompatibel ist mit einer Flexibilisierung von Versorgungs- und Angebotsstrukturen.

7) Arzneimittel- und Apothekenrecht werden angepasst, namentlich um auf rechtssicherer Basis erweiterte Abgabemöglichkeiten von Arzneimitteln durch
Vertragsärzte und Krankenhäuser zu schaffen im Rahmen von entsprechenden Vereinbarungen mit Krankenkassen. Das (aufgeweichte) Mehrbesitzverbot bei Apotheken wird insgesamt aufgehoben, um die bestehenden, eher an Zunftordnungen des ausgehenden achtzehnten als an modernen Dienstleistungserfordernissen des einundzwanzigsten Jahrhunderts ausgerichteten, Angebotsstrukturen zugunsten von effizienten Betriebs- und Vertriebsformen abzulösen.

Im Kontext der allenthalben öffentlich vorherrschenden Diskussionen und Positionierungen bezogen auf eine wettbewerblich organisierte Versorgungsgestaltung über die verschiedenen Leistungssegmente hinweg, mag die skizzierte umfassende Verantwortungs- und Kompetenzzuschreibung an die Krankenkassen zunächst sehr weitgehend erscheinen. Betrachtet man jedoch die Optionen im Licht der Notwendigkeit einer einheitlichen Versorgungsverantwortung realistisch, erscheint sie geradezu zwangsläufig.

\section{Bundesagentur vs. Pluralismus}

Die Option umfassender unmittelbarer staatlicher Steuerung wie in dezidiert staatlichen Gesundheitssystemen wäre eine theoretisch begründbare Alternative, um die aufgezeigten Defizite anzugehen. Die Implikationen wären jedoch außerordentlich weitreichend, beginnend mit der Notwendigkeit neuer Abgrenzungen von Bundes- und Landeskompetenzen, quasi Abschaffung der Krankenkassen und Umwidmung in eine einheitliche Struktur nach dem Muster einer „Bundesagentur für Gesundheit“ inklusiv verschiedenen regionaler „Gesundheitsagenturen“, die die Versorgung vor Ort im Sinne von Managementgesellschaften zu organisieren hätten. Der Wettbewerb auf der Versicherungsseite wäre damit negiert, auf der Leistungsanbieterseite potentiell sogar erhöht, da einer vielfältigen Angebotsstruktur ein Nachfragemonopolist gegenüber stünde. $\mathrm{Ob}$ allerdings staatliche Institutionen die Kraft für versorgungsrationale Einzelentscheidungen im Gesundheitswesen aufbringen, kann bereits mit Blick auf Entscheidungsprozesse zu Leistungsumfang oder Leistungsstandorten (z.B. in der Krankenhausplanung) mit Fug und Recht bezweifelt werden. Nicht umsonst 
ist die deutsche Gesundheitspolitik seit Jahrzehnten im Wesentlichen von einer Korporatisierungsstrategie geprägt, die viele Entscheidungen aus der Tagespolitik auslagert - unbeschadet davon, dass die korporatistischen Strukturen durch zunehmende staatliche Eingriffe in Teilen mittlerweile stark gelenkt oder insbesondere auf der Finanzierungs- bzw. Einnahmenseite unmittelbar durch staatliche Regulierung und Behörden (BVA) abgelöst wurden (vgl. etwa Pressel 2012, Noweski 2004).

Nach politischen Ansätzen, die einzelnen Krankenkassen stärker in einer Rolle als Versorgungsgestalter zu etablieren, erfolgte spätestens mit dem Versorgungsstrukturgesetz eine (vorübergehende?) Restauration der zentralistisch korporatistischen Strukturen (vgl. etwa Hermann 2011). Der Preis dafür bildet die weitere Verkrustung der Strukturen, die den eingangs skizzierten externen Anpassungsdruck immer ungenügender umsetzen können. Konfliktlösungsmechanismen fanden und finden demzufolge eher in Form zusätzlicher Finanzmittel denn in Strukturanpassungen statt. Insofern bieten die üblichen hinlänglich bekannten kleinen Eingriffe in das Machtgefüge zwischen den korporatistischen Institutionen bzw. zwischen diesen und unmittelbar staatlicher Kompetenzen wohl auch wenig Grund zur Hoffnung, damit letztlich den Anforderungen einer strukturierten Versorgung tatsächlich gerecht werden zu können. Schon im Jahr 2010 hat etwa die KBV (sic!) öffentlich das Scheitern der zentralistisch geplanten Honorarsystematik verkündet und eingestanden, dass deren Weiterführung „nur noch ins Chaos führen kann" (Köhler in ÄZonline, 30.07.2010). Freilich wurde daraus - wie schon so oft davor - nur der Schluss gezogen, dass man eben wieder eine neue Honorarsystematik benötige. Zur Hinwendung in Richtung auf grundsätzlichere Veränderungen reichte es auch 2010 wieder einmal nicht.

\section{AOK Baden-Württemberg als Player}

Es verbleiben somit grundsätzlich die Krankenkassen als strukturelle Player und die Frage, ob sie diesem Anspruch gerecht werden können. Im Bereich der Selektivverträge und der zielgenauen Leistungssteuerung liegen auf der Seite von Krankenkassen Erfahrungen hinsichtlich Versorgungsgestaltung vor. Die entsprechenden Erfahrungen und Ergebnisse aus Sicht der AOK BadenWürttemberg werden im Folgenden dargestellt. Dabei erfolgt eine Fokussierung auf die hausarztzentrierte Versorgung nach $\mathbb{} 73$ b SGB V $(\mathrm{HZV})$, da sie zum einen den umfassendsten strategischen und prozessualen selektivvertraglichen Ansatz der AOK Baden-Württemberg (und derzeit wohl insgesamt in der GKV) abbildet und darüber hinaus zu weiterführenden Implikationen für eine funktionale Ausgestaltung eines wettbewerblichen Ordnungsrahmens im Sinne der Themenstellung hinleitet.

\subsection{Die HZV-Strategie}

Die HZV-Strategie der AOK BadenWürttemberg folgt der Überzeugung, dass eine flächendeckende, für alle Bürger zugängliche hausärztliche Versorgung das Rückgrat eines modernen, leistungsfähigen Gesundheitssystems darstellt. Hausärzte sind in der Regel

\section{Eine flächendeckende, für alle zugängliche hausärztliche Versorgung stellt das Rückgrat eines modernen, leistungsfähigen Gesundheitssystems dar.}

die erste medizinische Anlaufstelle für Menschen mit gesundheitlichen Problemen und sie beurteilen, ob weitere Behandlungsschritte, wie beispielsweise die Überweisung zu einem Facharzt oder eine stationäre Aufnahme, erforderlich sind. Besondere Bedeutung kommt der hausärztlichen Versorgung im Management der Volkskrankheiten $\mathrm{zu}$, wie es sich etwa in den DiseaseManagement-Programmen als explizit hausarztbasierten Versorgungskonzepten widerspiegelt. Diese Überzeugung der AOK Baden-Württemberg steht in breiter Übereinstimmung mit den aktuellen DEGAM-Zukunftspositionen (DEGAM 2012).

Trotz der elementaren Bedeutung einer leistungsfähigen allgemeinmedizinischen Versorgungsebene hat diese in den letzten Jahrzehnten relativ an Gewicht gegenüber Spezialisierungen verloren.
Der Gesetzgeber versucht seit langem diesem Trend entgegenzuwirken, allerdings nur mit begrenztem Erfolg. Die AOK Baden-Württemberg erkannte die Chancen der hausarztzentrierten Versorgung frühzeitig und schloss im Mai 2008 als bundesweit erste Krankenkasse einen Hausarztvertrag mit Vollversorgungsanspruch und Bereinigung ab.

Die im Vertrag festgelegte deutlich pauschalierte Vergütungssystematik mit wenigen Einzelleistungen, qualitätsund ergebnisabhängigen Zuschlägen, einem Zuschlag für chronisch Kranke und der Wegfall von fallzahlorientierten Mengenbegrenzungen stellen einen Paradigmenwechsel dar, der die vom Sachverständigenrat 2009 dargelegten Empfehlungen in weiten Teilen umsetzt. Die Vertragsstruktur unterstützt insbesondere große Versorgerpraxen mit klassischem breitem hausärztlichen Behandlungsspektrum, so dass sich im Ergebnis zum einen überdurchschnittlich viele große Praxen und zum anderen überdurchschnittlich viele Praxen in sog. ländlichen Räumen an dem Vertrag beteiligen. Flankiert wird die Vertragsstruktur durch zahlreiche unterstützende Elemente wie eine neue IT-Struktur, die das Handeln im Sinne der Vertragsziele unterstützt oder etwa durch die über 300 etablierten und von der AOK finanzierten Qualitätszirkel zur rationalen Pharmakotherapie. Des Weiteren setzt der Vertrag finanzielle Anreize zur Beschäftigung von speziell weitergebildeten Versorgungsassistentinnen in der Hausarztpraxis (VERAH). Damit befördert die hausarztzentrierte Versorgung der AOK Baden-Württemberg bewusst einen Entwicklungspfad in den Hausarztpraxen hin zu professionellen Betreuungsstrukturen für chronisch Kranke im Rahmen von Teamstrukturen und ärztlicher sowie nichtärztlicher Arbeitsteilung. Neben einer Intensivierung von vorausschauender Behandlungsplanung und Strukturierung der Versorgung unmittelbar aus Patientensicht geht damit auch eine Entlastung der zunächst knapper werdenden hausärztlichen Ressourcen einher. Bereits heute findet mit universitären Partnern oder innovativen Dienstleitern eine kontinuierliche Weiterentwicklung von Versorgungsstrukturen in der HZV mit neuen Ansätzen statt, etwa ein hausarztbasiertes 
Case-Management von multimorbiden Patienten oder die Unterstützung von empfohlenen Lebensstilveränderungen unter Nutzung neuer Medien (siehe z.B. Gesundheit + Gesellschaft 06/12) .

\subsection{Die HZV-Evaluation}

Um die Wirkungen des AOK-Hausarztvertrages zu messen, beauftragten die Vertragspartner das Institut für Allgemeinmedizin der Universität Frankfurt am Main (Prof. Gerlach) und die Abteilung für Allgemeinmedizin und Versorgungsforschung der Universitätsklinik Heidelberg (Prof. Szecsenyi) mit der wissenschaftlichen Evaluation (vgl. www. hzv-aktuell.de)

Eine insbesondere aus versorgungspolitischer Sicht spannende Frage ist, ob es den teilnehmenden Hausärzten tatsächlich gelingt, mehr Verantwortung für chronisch Kranke zu übernehmen und deren Versorgung besser zu strukturieren und zu koordinieren. Die Ergebnisse sprechen eindeutig dafür: Die am Hausarztvertrag teilnehmenden Versicherten sind zum einen älter und kränker als die Versicherten in der Regelversorgung, zum anderen werden sie intensiver betreut. So hat ein im Hausarztvertrag eingeschriebener Versicherter im Halbjahr durchschnittlich zwei Arztkontakte mehr als ein Versicherter vergleichbarer Morbidität in der Regelversorgung. Gleichzeitig nahm die Zahl der (unkoordinierten) Facharztbesuche deutlich ab. Durch die damit verbundene Entlastung der Fachärzte werden Kapazitäten für medizinisch notwendige Termine freigesetzt. Die in den Qualitätszirkeln vermittelten Inhalte zur Arzneimitteltherapie zeigen ebenfalls Wirkung: Die Zahl der Verordnungen von Medikamenten ohne therapeutischen Zusatznutzen und die Anzahl der Patienten, die regelmäßig fünf oder mehr Medikamente einnehmen und dadurch besonders durch Arzneimittelwechselwirkungen gefährdet sind, entwickelt sich günstiger als in der Regelversorgung. Des Weiteren erzielt die HZV eine deutlich höhere Umsetzungsquote von Rabattarzneimitteln, was in hohem Maße zur ökonomisch ausgeglichenen Vertragsumsetzung beiträgt.

Deutlich über 1.000 VERAHs, die inzwischen in rund einem Drittel der HZV-Praxen beschäftigt sind, übernehmen vom Medikamenten- und Wund- management bis hin zu Hausbesuchen wichtige Management- und Unterstützungsfunktionen und optimieren so die Versorgung von chronisch kranken $\mathrm{Pa}$ tienten. Die große Mehrheit der VERAHs und Hausärzte ist einhellig der Meinung, dass sich die Patientenversorgung durch die Tätigkeit der VERAH verbessert hat und der Arzt spürbar entlastet wird. Die Befragung von über 750 Hausärzten zeigte außerdem, dass HZV-Ärzte trotz höherer Arbeitsbelastung zufriedener mit ihrem Einkommen sind, sich weniger gestresst fühlen und motivierter sind, Veränderungsprozesse anzustoßen. Damit leistet die HZV einen wichtigen Beitrag zur Steigerung der Attraktivität des Hausarztberufs sowie zu dem vom SVR empfohlenen Umbau von Versorgungsstrukturen, die das Gesundheitssystem auf die demographischen Herausforderungen und die Entwicklungen in der ärztlichen Berufsausübung einstellen.

\subsection{HZV und Facharztverknüpfungen}

Die HZV stellt somit die Basis einer effektiven und effizienten Versorgungssteuerung auf der Grundlage einer klaren Patientenbindung dar. Strukturierung bedingt Verbindlichkeit im Sinne eines wechselseitigen Committments und Vertrauens zwischen (Haus-)Arzt und Patient einerseits und im Weiteren zwischen Versicherten bzw. Patient und seiner Krankenkasse und deren Versorgungsangebot andererseits. Auf diesem Grundverständnis einer rationalen Versorgungsstrukturierung basieren die weiteren an die HZV angefügten Angebote und Versorgungspfade inhaltlich und qualitativ. Anknüpfungspunkte einer strukturierten Versorgung sind z.B. unmittelbare Angebote der AOK wie deren Gesundheitsangebote oder Beratungsangebote des Sozialen Dienstes zur Herstellung von stabilen Gesamtversorgungssituationen.

Ein weiterer Anknüpfungspunkt sind Verträge zur besonderen ambulanten ärztlichen Versorgung nach $\mathbb{S} 73 \mathrm{c}$ SGB V. Die AOK Baden-Württemberg hat bislang 73c-Verträge in den Bereichen Kardiologie, Gastroenterologie, Psychotherapie, Psychiatrie und Neurologie geschlossen. Allen Verträgen ist gemeinsam, dass sie die für die jeweiligen Fachgruppen relevanten medizinischen Versorgungsbereiche anhand der qualitativ besten verfügbaren Leitlinien definieren und die Vergütungsstruktur sich vor allem an den Krankheitsbildern und nicht an einzelnen Verrichtungen ausrichtet. Damit werden ein verbindlicher Indikationsbezug des Handelns und somit gleichzeitig eine sorgfältige Diagnosestellung als Ausgangspunkt aller weiteren Therapieoptionen befördert. Wesentliches Merkmal der 73cVertragsgestaltung ist ferner, dass an diesen Verträgen nur Versicherte der AOK Baden-Württemberg teilnehmen können, die bereits in der HZV eingeschrieben sind. Denn HZV und 73cVerträge sind strukturell miteinander verschränkt hinsichtlich inhaltlicher Therapiepfade und Schnittstellen der ärztlichen Tätigkeiten zwischen den Beteiligten sowie einheitlicher Empfehlungen etwa im Arzneimittelmanagement. Die Weiterführung der spezifisch an Qualitätskriterien und gemeinsamem Kooperationsverständnis ausgerichteten Versorgungspfade in viele weitere Leistungsfelder liegt auf der Hand, bedarf jedoch der aktiven Gestaltung und - um schon allein dem Anschein der wechselseitigen Vorteilnahme („Zuweisung gegen Entgelt“) die Grundlage zu entziehen -, stets der Legitimität der vertrag-

Die HZV leistet einen wichtigen Beitrag zur Steigerung der Attraktivität des Hausarztberufs und zum Umbau der Versorgungsstrukturen.

lichen Gestaltung über Krankenkassen (vgl. BT-Drucks. 17/6906, S.55). Auch hinsichtlich der Kommunikation und Erläuterung der Vertragsinhalte und deren Umsetzung beschreitet die AOK Baden-Württemberg mit dem kassenseitigen Arztpartner-Service (APS) neue Wege. Die dezentral in ganz Baden-Württemberg aufgestellten Mitarbeiterinnen und Mitarbeiter stehen den teilnehmenden Praxen als Berater, Kümmerer und „Vernetzer“ zur Verfügung. Sie leisten über persönliche Kommunikation vor Ort ihren Beitrag zu einem partnerschaftlichen Miteinander und 
einer erfolgreichen Vertragsumsetzung.

\section{Stillstand als System(erhaltungsstrategie)}

Als wesentlicher Hemmschuh einer schnelleren und intensiveren Umsetzung von besser strukturierten und integrierten Versorgungskonzepten auf vertraglicher Basis erweist sich immer wieder eine generelle Systemträgheit, die ihre Ursachen teilweise auch in einer finanziellen Saturiertheit der Beteiligten zu haben scheint, die sich aus den eingangs skizzierten primär finanziell dominierten dysfunktionalen Handlungsstrategien in zentralistisch gesteuerten verkrusteten Strukturen ableiten lässt.

Die bislang erzielten Erfolge selektivvertraglicher Versorgungsgestaltung beruhen auf einer konstruktiv vertrauensvollen Zusammenarbeit der Vertragspartner und erfordern so viel Geduld wie Ausdauer als auch - wettbewerblich motivierte - Innovationsfreude. Von hoher Bedeutung ist dabei die unmittelbar gelebte Verantwortungsübernahme für die regionale Versorgung durch die AOK Baden-Württemberg im Wege der Übernahme des Sicherstellungsauftrages im Rahmen von HZV und 73c-Verträgen gemeinsam mit den Partnern Hausärzteverband und MEDI sowie verschiedenen unterstützenden Berufsverbänden.

Dies scheint zwischenzeitlich auch die KBV registriert zu haben und sinniert derzeit prominent öffentlich darüber, ob der Sicherstellungsauftrag nicht generell besser bei den Krankenkassen anzusiedeln wäre (Köhler 2012). Die in diesem Zusammenhang von dort aufgestellten Forderungen für das Festhalten der KVen am Sicherstellungsauftrag orientieren sich indessen an einer rückwärts gewandten Lagerfeuerromantik für Ärzte, passen jedoch nicht in eine strukturierte Versorgungswelt von heute und morgen. $\mathrm{Zu}$ solchen Forderungen gehören etwa der Wunsch nach einer Wiederherstellung der diagnostischen und therapeutischen „Freiheit“ (welche Funktion haben dann Leitlinien, GBA, evidenzbasierte Medizin und Qualitätsvorgaben?), die Abschaffungen jeglicher Mengensteuerung und Bezahlung aller (Einzel-) Leistungen mit festen Preisen (entsprechende historische Erfahrungen zur Auswirkung liegen vor und werden verhindern, dass dies je so kommen wird), Abschaffung von Regressen bei veranlassten Leistungen (da müssten zunächst wirksame alternative Steuerungskonzepte vorgelegt werden, die einen ökonomische Einsatz der Mittel verlässlich bewirken) und schließlich die Zurückdrängung von institutionellen Öffnungen der Krankenhäuser für ambulante Behandlungen (ein Trend, der weder wettbewerblich noch versorgungspolitisch noch versorgungsökonomisch umkehrbar sein wird). Im Kern gehen diese Forderungen an der notwendigen Versorgungsstrukturierung völlig vorbei, sie bleiben im Wesentlichen dem materiellen Wunschdenken und angeblich schrankenloser freier Berufsausübung einer untergegangenen Epoche verhaftet, ohne realistische Alternativen für eine Gesellschaft des langen Lebens aufzuzeigen.

Die von der AOK Baden-Württemberg gemeinsam mit regionalen Vertragspartnern aufgebaute Grundstruktur bietet dagegen vielfältige Anknüpfungspunkte für die weitere Entwicklung von leistungsfähigen Praxen und Vernetzungsoptionen zu weiterführenden Versorgungsstrukturen. Im Ergebnis sollten Krankenkassen mit der Übernahme des Sicherstellungsauftrages nicht so hasenfüßig umgehen.

\section{Fazit und pragmatischer Einstieg}

Auch vor diesem Hintergrund stellt der durch das GKV-FinG eingeführte Investitionsvorbehalt in $\mathbb{S} 73 \mathrm{~b}$ Abs. 5a SGB V eine Gefährdung für die Weiterentwicklung nachweislich erfolgreicher hausarztzentrierter und darüber hinausweisender Versorgungsstrukturen dar. Er sollte ersatzlos gestrichen werden, da er Innovation über Investitionen verhindert. Zielführend wäre es im Weiteren, die skizzierten sieben Reformpunkte gesetzgeberisch aufzugreifen, um das Potential wettbewerblicher Suchprozesse für optimierte Versorgungsprozesse zu erschließen.

Einen pragmatischen Einstieg für einen fließenderen Übergang in eine breitere Verantwortungsübernahme durch Krankenkassen würde ein 2-Säulen-Modell darstellen, das neben einem anzupassenden Kollektivvertragssystem (s.o.) für Krankenkassen ein Optionsmodell eröffnen würde, demzufolge sie zunächst für in Selektivverträgen eingebundene Versicherte insbesondere die Kontrahierungszwänge im Bereich fachärztlicher, spezialfachärztlicher und bestimmter stationärer Leistungen lösen können. Das entsprechende Versichertenkollektiv könnte konkret der Versichertengruppe entsprechen, die sich freiwillig über die hausarztzentrierte Versorgung hinaus in ein Gesamtversorgungsmodell ihrer Krankenkasse begibt. Für diese Ver-

Krankenkassen sollten mit der Übernahme des Sicherstellungsauftrages nicht hasenfüßig umgehen.

sicherten unterliegt die Krankenkasse keinem der kollektivvertraglichen Uniformitätszwänge und kann für ihre Versicherten ein qualitativ hochattraktives Angebot offerieren. Aufgrund der Freiwilligkeit und des vorauszusetzenden Konsenses zur Gestaltung des Gesamtversorgungsmodells insbesondere mit den hausärztlichen Partnern der HZV wäre sichergestellt, dass Koordination, Kooperation und Qualität im Sinne der eingangs skizzierten physischen Produktionseffizienz zu den Fixpunkten einer entsprechenden Versorgungsgestaltung würden.

\section{Literatur:}

Cassel, D/ Ebsen, I./ Greß, S./ Jacobs, K./ Schulze, S./ Wasem, J. (2008): Vertragswettbewerb in der GKV, Bonn, WidO, S. 219-220. DEGAM (2012): Allgemeinmedizin - spezialisiert auf den ganzen Menschen. Positionen zur Zukunft der Allgemeinmedizin und hausärztlichen Praxis, frei zugänglich unter www.degam.de.

Drösler, S. et al. (2011): Evaluationsbericht zum Jahresausgleich 2009 im Risikostruktur ausgleich

Graf,J. (2009): Vertragswettbewerb brauch faire Bereinigungsregeln. Monitor Versorgungsforschung 2 (06):36-38.

Greß, S. (2009): Mit gleichen Rahmenbedingungen zu einem fairen Wettbewerb im Gesundheitssystem, WISO direkt Mai 2009, Bonn, Friedrich-Ebert-Stiftung.

Hermann, C. (2011): Weihnachtsinterview. In: Highlights - Fakten und Hintergründe, 8 Jahrgang 2011,30/11.

Köhler, A. (2012): Bericht an die Vertreterversammlung - Rede des Vorsitzenden des KBV Vorstandes Dr. Andreas Köhler, 28.9.2012. 
Leber, W.-D./ Malzahn, J./ Wolff, J. (2007): Neuer Rahmen für die Kliniken. In: Gesundheit und Gesellschaft, Kompart-Verlag, 10. Jahrgang, Ausgabe 7-8/07, S. 27-31.

Noweski, M. (2004): Der unvollendete Korporatismus - Staatliche Steuerungsfähigkeit im ambulanten Sektor des deutschen Gesundheitswesens, Berlin, WZB.

Pressel, H (2012): Der Gesundheitsfonds Entsteheung/ Einführung/ Weiterentwicklung/ Folgen, Heidelberg, Springer VS.

Schönbach, K.-H. (2011): Sektorübergreifende Sicherstellung mit Vertragspflicht der Krankenkassen. In: Jacobs, K. / Schulze, S. (Hrsg): Sicherstellung der Gesundheitsversorgung - Neue Konzepte für Stadt und Land, Bonn, WidO; S. 97-115

SVR, Gutachten 2000/2001: Bedarfsgerechtigkeit und Wirtschaftlichkeit.

SVR, Gutachten 2005: Koordination und Qualität im Gesundheitswesen.

SVR, Gutachten 2007: Kooperation und Verantwortung - Voraussetzungen einer zielorientierten Gesundheitsversorgung.

SVR, Sondergutachten 2009: Koordination und Integration - Gesundheitsversorgung in einer Gesellschaft des längeren Lebens.

SVR, Sondergutachten 2012: Wettbewerb an der Schnittstelle zwischen ambulanter und stationärer Gesundheitsversorgung.

\section{Das Recht auf Nichtwissen.}

Möglichkeiten der Erweiterung des Neugeborenenscreenings als prädiktiver Reihenuntersuchung?

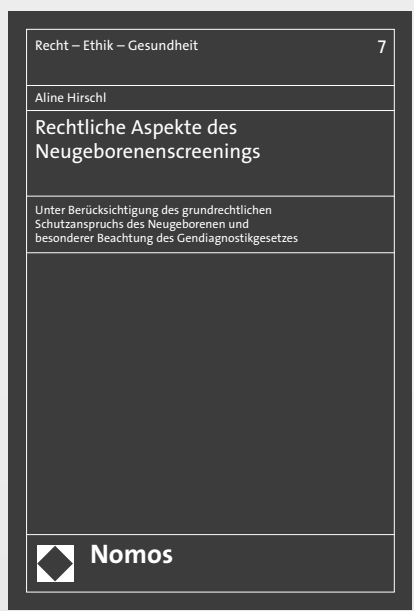

\footnotetext{
Rechtliche Aspekte des Neugeborenenscreenings Unter Berücksichtigung des grundrechtlichen Schutzanspruchs des Neugeborenen und besonderer Beachtung des Gendiagnostikgesetzes Von Aline Hirschl 2013, 346 S., brosch., 89,- $€$ ISBN 978-3-8487-0113-1

(Recht-Ethik-Gesundheit, Bd. 7)
}

Prädiktive Reihenuntersuchungen an Neugeborenen auf behandelbare Krankheiten sind etabliert - aber wäre auch eine Ausweitung auf unbehandelbare oder spätmanifestierende Krankheiten zulässig? Das Buch widmet sich unter Einbeziehung der medizinischen Erkenntnisse den Vorgaben des Verfassungsrechts und den bestehenden einfachgesetzlichen Regelungen. 